\title{
Interplay between symmetry and spin-orbit coupling on graphene nanoribbons
}

\author{
Hernán Santos, ${ }^{1}$ M. C. Muñoz, ${ }^{2}$ M. P. López-Sancho, ${ }^{2}$ and Leonor Chico $^{2}$ \\ ${ }^{1}$ Departamento de Física Fundamental, Universidad Nacional de Educación a Distancia, Apartado 60141, E-28080 Madrid, Spain \\ ${ }^{2}$ Instituto de Ciencia de Materiales de Madrid, Consejo Superior de Investigaciones Científicas, Sor Juana Inés de la Cruz 3 , \\ 28049 Madrid, Spain
}

(Received 26 February 2013; published 3 June 2013)

\begin{abstract}
We study the electronic structure of chiral and achiral graphene nanoribbons with symmetric edges, including curvature and spin-orbit effects. Curved ribbons show spin-split bands, whereas flat ribbons present spin-degenerate bands. We show that this effect is due to the breaking of spatial inversion symmetry in curved graphene nanoribbons, while flat ribbons with symmetric edges possess an inversion center, regardless of their having chiral or achiral edges. We find an enhanced edge-edge coupling and a substantial gap in narrow chiral nanoribbons, which is not present in zigzag ribbons of similar width. We attribute these size effects to the mixing of the sublattices imposed by the edge geometry, yielding a behavior of chiral ribbons that is distinct from those with pure zigzag edges.
\end{abstract}

DOI: 10.1103/PhysRevB.87.235402

PACS number(s): 71.20.Tx, 73.22.-f, 71.70.Ej

\section{INTRODUCTION}

The crucial interplay between structure and electronic properties of graphene is among the most attractive features of its derived nanomaterials. Both carbon nanotubes (CNTs) and graphene nanoribbons (GNRs) show promising characteristics for spintronic devices. Recent progress in experimental techniques has allowed for the fabrication of graphene nanostripes by using electron-beam lithography ${ }^{1}$ or by unrolling CNTs. ${ }^{2}$ These ribbons could be used in electronic devices, such as field-effect transistors, ${ }^{3}$ opening new perspectives for nanoelectronics.

The presence of localized edge states in GNRs, theoretically predicted, ${ }^{4,5}$ and experimentally proved, ${ }^{6}$ confers them distinct properties. GNRs have attracted a great amount of theoretical work but mostly focused on high-symmetry zigzag and armchair achiral ribbons. Zigzag ribbons have edges states with different spin polarizations, while armchair nanoribbons do not have edge states. The edges of minimal ${ }^{7,8}$ chiral ribbons can be considered as a mixture of armchair and zigzag edges, thus having edge-localized states stemming from their zigzag part. Although the evolution of the nanoribbon band structure upon the change of chirality has been recently addressed, ${ }^{9-11}$ these systems have been nonetheless much less studied and many aspects remain to be explored.

The seminal work of Kane and Mele ${ }^{12}$ triggered the interest on new quantum phases of matter and on the spin-orbit coupling (SOC) effect, which, although known to be small in graphene, gives rise to important physics. In particular, the quantum spin Hall (QSH) phase has been widely addressed. ${ }^{13,14}$

Curvature is known to enhance spin-orbit interaction; its importance in SOC effects has been theoretically investigated for the honeycomb lattice, especially for CNTs, ${ }^{15-20}$ and experimentally confirmed. ${ }^{21}$ Hybridization of $\pi$ and $\sigma$ orbitals, decoupled in flat graphene, is enhanced by curvature and thus SOC effects are bolstered. ${ }^{18}$ The interplay between curvature and SOC in GNRs has been mostly focused in achiral ribbons, with highly symmetric zigzag and armchair edges. ${ }^{22-24}$ For zigzag GNRs, dispersionless edge bands in the flat geometry were found to become dispersive because of SOC effects.
Both $\pi$ and $\sigma$ edge states remain spin filtered in the curved geometry, still localized at the boundaries of the ribbon, albeit with an in-plane spin component and a localization length larger than for the flat case. ${ }^{23}$ Recent experiments ${ }^{2,25,26}$ on chiral GNRs obtained by unzipping CNTs show a reminiscent curvature. Scanning tunneling spectroscopy measurements revealed the presence of one-dimensional edge states, with an energy splitting dependent on the width of the ribbon. ${ }^{26}$ By comparison with calculations employing a $\pi$-band model with a Hubbard term the width dependence of the edge state gap was interpreted as a consequence of spin-polarized edge states. ${ }^{11,26}$ Hence, the study of curvature effects in GNRs is relevant from the experimental and theoretical viewpoint.

In this paper we address the study of this ampler class of ribbons with chiral edges, focusing on the differences of SOC effects in flat and curved nanoribbons. We summarize our main results as follows:

(i) We find that the bands of both chiral and achiral flat ribbons with symmetric edges are at least twofold spin degenerate due to spatial inversion symmetry. Curving the ribbons breaks this symmetry, thus yielding spin-split bands except for the time-reversal protected special symmetry $k$ points.

(ii) We find a gap in all chiral ribbons, despite the fact that they have a zigzag edge component. Boundary conditions in chiral ribbons mix both sublattices at each edge. This enhances edge-edge coupling, which results in a substantial gap without invoking electron interactions.

(iii) Curvature augments spin-orbit effects in GNRs, yielding a larger splitting in the spin-split bands. In fact, curvature may induce metallicity in ribbons which have a gap in the planar form.

(iv) The spatial distribution of edge states depends on curvature and chirality. While zigzag ribbons are known to have spin-filtered states at the edges, in narrow $(\approx 40 \AA)$ chiral ribbons edge states can have nonzero density at both edges, due to the edge-edge coupling. This size effect is more evident in ribbons with chiral angle close to $30^{\circ}$, i.e., that of the armchair edge, for which the sublattice mixing is stronger.

This paper is outlined as follows. Section II describes the structure and geometry of the ribbons studied. Section III gives 
some symmetry considerations concerning the role of spatial inversion in flat and curved general ribbons. Section IV contains the description of the model Hamiltonian and calculation method. Section $\mathrm{V}$ presents the results, including spin-orbit interaction, for ribbons of different widths in flat and curved geometries. Finally, in Sec. VI we discuss our results and final conclusions are drawn.

\section{GEOMETRY}

We focus on chiral ribbons with symmetric minimal edges, ${ }^{7}$ obtained from unrolling chiral carbon nanotubes. The ribbon is thus characterized by the edge vector $\boldsymbol{T}=n \boldsymbol{a}_{1}+m \boldsymbol{a}_{2}$, where $\boldsymbol{a}_{1}$ and $\boldsymbol{a}_{2}$ are the primitive vectors of graphene, and the width vector $\boldsymbol{W}$. The widths considered are therefore given by an integer multiple of $\boldsymbol{H}$, defined as the smallest graphene lattice vector perpendicular to $\boldsymbol{T}$, as depicted in Fig. 1. For a given $\boldsymbol{T}, \boldsymbol{H}$ is uniquely determined up to a global \pm 1 factor. As $\boldsymbol{W}=\boldsymbol{M} \boldsymbol{H}$, we will denote the ribbons by $M(n, m)$, where $M$ states the width of the ribbon and $(n, m)$ indicates the minimal edge. All minimal edges can be decomposed in a zigzag and an armchair part, ${ }^{7,8} \boldsymbol{T}=n_{Z} \boldsymbol{T}_{Z}+n_{A} \boldsymbol{T}_{A}$, with $\boldsymbol{T}_{Z}=\boldsymbol{a}_{1}$ and $\boldsymbol{T}_{A}=\boldsymbol{a}_{1}+\boldsymbol{a}_{2}$.

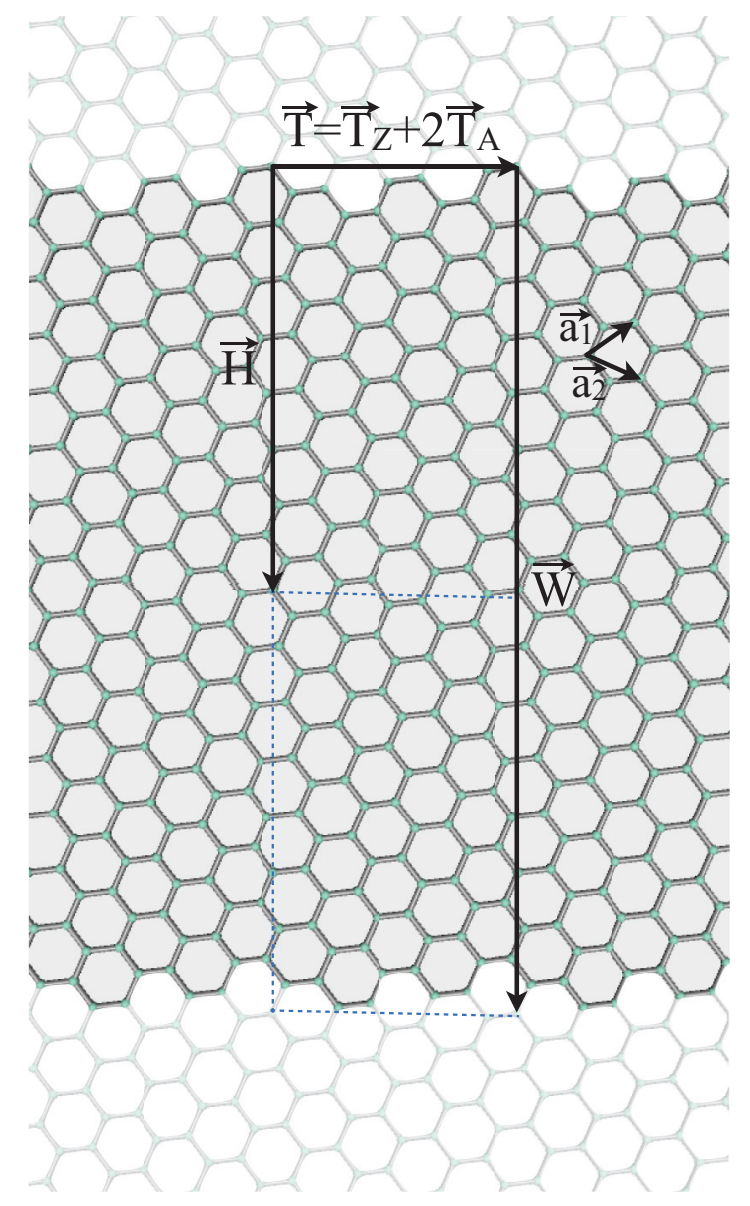

FIG. 1. (Color online) Geometry of the 2(3,2) GNR highlighted in dark gray on a graphene sheet, showing its translation vector $\boldsymbol{T}=$ $\boldsymbol{T}_{Z}+2 \boldsymbol{T}_{A}$ and its width vector $\boldsymbol{W}=2 \boldsymbol{H}$, where $\boldsymbol{H}=-7 \boldsymbol{a}_{1}+1 \boldsymbol{a}_{2}$. The unit cells spanned by $\boldsymbol{T}$ and $\boldsymbol{H}$ or $\boldsymbol{W}$ are indicated with dotted lines.
The chirality of the ribbon is specified by the chiral angle $\theta$ between the translation vector $(n, m)$ which defines the edges and the zigzag direction $(1,0)$.

We take into account different curvatures in the transversal direction for a given flat GNR, with no stretching allowed. Curvature is denoted by the angle $\varphi$ spanned by the ribbon from its curvature center, ranging from zero for a flat ribbon to a value of $2 \pi$, which corresponds to a nanotube with cut bonds along its length. The degree of curvature is controlled by the angle and by the diameter of the cylindrical configuration.

\section{SYMMETRY CONSIDERATIONS}

Carbon nanotubes are classified as achiral and chiral according to their having a symmorphic or non-symmorphic symmetry group, respectively. This means that chiral tubes possess a spiral symmetry, so that there are two enantiomers for each chirality, while achiral tubes are equal to their mirror image; i.e., achiral tubes present space inversion symmetry while chiral tubes do not. ${ }^{27,28}$ Graphene nanoribbons, like their siblings carbon nanotubes, are customarily classified as achiral and chiral according to their edge shapes. In GNRs this classification is related to the crystallographic orientation of the boundaries: ribbons with zigzag and armchair edges (derived from armchair and zigzag CNTs, respectively) are called achiral, and those obtained from chiral tubes are called chiral GNRs. However, these so-called chiral ribbons with symmetric edges do have an inversion center. Upon bending the ribbon the inversion symmetry is lost. This feature is crucial when considering SOC effects. Figure 2 shows an example of a flat (left panel) and curved (right panel) unit cell of the $(3,2)$ ribbon, the latter with $\varphi=\pi$. A symmetry center is indicated in the planar geometry. No such inversion center exists in the curved nanoribbon.

Notice that a flat nanoribbon with different edges lacks inversion symmetry. This situation is of experimental interest: most likely, actual ribbons will not have symmetric edges. Such asymmetry can be achieved either by adding or removing atoms to an originally edge-symmetric ribbon, or by altering the bond lengths in one of the edges. Dissimilar bond lengths
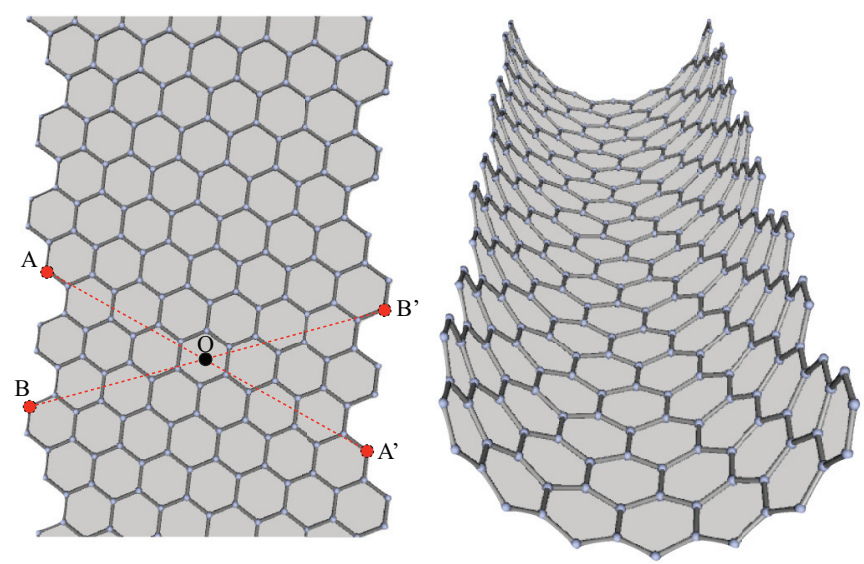

FIG. 2. (Color online) Schematic geometry of a flat (left) and curved (right) $1(3,2)$ GNR. The curvature angle is $\varphi=\pi$. Two pairs of equivalent atoms under spatial inversion symmetry are highlighted, and the inversion center is marked as $O$. 
may arise as a result of a different functionalization on the two edges of the nanoribbon.

\section{THEORETICAL MODEL AND COMPUTATIONAL METHODS}

We calculate the band structure of graphene within the empirical tight-binding (ETB) approximation. Although the $\pi$-orbital tight-binding model is known to capture the lowenergy physics of graphene, since we are interested in SOC effects we consider here an orthogonal four-orbital $2 s, 2 p_{x}$, $2 p_{y}, 2 p_{z}$ basis set. This allows for the inclusion of the intrinsic SO terms within the conventional on-site approach. The matrix Hamiltonian is built following the Slater-Koster formalism up to nearest-neighbor hopping. We use the parametrization obtained by Tománek-Louie for graphite. ${ }^{29}$ The expression of the one-electron ETB Hamiltonian is

$$
H_{0}=\sum_{i, \alpha, s} \epsilon_{\alpha}+\sum_{\langle i, j\rangle, \beta, s} t_{i j}^{\alpha, \beta} c_{i, s}^{\alpha+} c_{j, s}^{\beta}+\text { H.c. },
$$

where $\epsilon_{\alpha}$ represents the atomic energy of the orbital $\alpha,\langle i, j\rangle$ stands for all the atomic sites of the unit cell of the GNR, and $c_{i, s}^{\alpha+}$ and $c_{i, s}^{\alpha}$ are the creation and annihilation operators, respectively, of one electron at site $i$, orbital $\alpha$, and spin $s$. We focus on neutral graphene; thus, no doping effects are addressed.

SOC effects are included by adding an atomiclike term $H_{\text {So }}$ to the $H_{0}$ Hamiltonian. Assuming that the most important contribution of the crystal potential to the spin-orbit coupling is close to the cores, the $H_{\text {SO }}$ contribution takes the form

$$
H_{\mathrm{SO}}=\sum_{i} \frac{\hbar}{4 m^{2} c^{2}} \frac{1}{r_{i}} \frac{d V_{i}}{d r_{i}} \boldsymbol{L} \cdot \boldsymbol{S}=\lambda \boldsymbol{L} \cdot \boldsymbol{S},
$$

where the spherical symmetry of the atomic potential $V_{i}$ has been assumed and $r_{i}$ is the radial coordinate with origin at the $i$ atom. $\boldsymbol{L}$ stands for the orbital angular momentum of the electron, and $\boldsymbol{S}$ is the spin operator. The parameter $\lambda$ is a renormalized atomic SOC constant, which depends on the orbital angular momentum. Notice that the $H_{\text {SO }}$ terms only couple $p$ orbitals in the same atom. Considering the spin parts of the wave functions, the Hamiltonian matrix has $8 N_{a} \times 8 N_{a}$ elements, $N_{a}$ being the number of the $\mathrm{C}$ atoms in the unit cell of the GNR and 8 corresponding to the four orbitals per spin of the $s p^{3}$ basis set. The total Hamiltonian in the $2 \times 2$ block spinor structure is given by

$$
H=\left(\begin{array}{cc}
H_{0}+\lambda L_{z} & \lambda\left(L_{x}-i L_{y}\right) \\
\lambda\left(L_{x}+i L_{y}\right) & H_{0}-\lambda L_{z}
\end{array}\right) .
$$

The total Hamiltonian $H$ incorporates both spin-conservation and spin-flip terms. The spin-conserving diagonal terms act as an effective Zeeman field producing gaps at the $K$ and $K^{\prime}$ points of the graphene Brillouin zone (BZ), with opposite signs. ${ }^{18}$ By exact diagonalization of the matrix $H$ we obtain the band structure of GNRs. As explained in the previous section, the curved geometry is obtained by isotropically bending the ribbon in the width direction, without changing the distance along its length. Thus, no bond stretching is included along the ribbon axis. We do not consider reconstruction or relaxation of the edges or passivation of the dangling bonds.
The value of the SOC constant for C-based materials is not well established and it is still under debate. Some theoretical estimates gave $\lambda=1 \mu \mathrm{eV}$ for graphene, ${ }^{30,31}$ much smaller than the atomic SO coupling, $8 \mathrm{meV}$. Taking into account the role of $d$ orbitals, this value rises to $\lambda=25 \mu \mathrm{eV}$. $^{32}$ Accurate measurements of SOC are difficult to perform in graphene because external effects such as substrates, electric fields, or impurities may mask its value. However, recent experiments in CNT quantum dots have reported spin-orbit splittings substantially higher than those theoretically predicted: Kuemmeth et $a .^{21}$ give a maximum splitting of $0.37 \mathrm{meV}$ in a CNT of diameter $5 \mathrm{~nm}$. One possible explanation for this energy splitting is that a higher value of $\lambda$ should be considered; as indicated by Izumida et al., ${ }^{19}$ those measurements are compatible with $\lambda=14 \mathrm{meV}$. More recently, Steele et al. ${ }^{33}$ have presented evidence of large spin-orbit coupling in CNTs, up to $3.4 \mathrm{meV}$, an order of magnitude larger than previously measured and the largest theoretical estimates. Furthermore, transport experiments report spin-relaxation times in graphene 1000 times lower than predicted. ${ }^{34}$ This is compatible with a larger value of the SOC coupling than those given by previous theoretical estimates. ${ }^{30-32}$ Although small, its effects in GNRs could have important consequences when considering the spin degree of freedom, as has been experimentally shown in CNTs. ${ }^{21,33}$ For the sake of clarity, we choose for the figures a spin-orbit interaction parameter $\lambda=0.2 \mathrm{eV}$.

The spin-orbit contribution to the Hamiltonian, $H_{\mathrm{SO}}$, is linear on $\lambda$. We have checked that, for small values of this parameter, such as the one employed here and those of physical relevance, the eigenvalues of the full Hamiltonian $H$ are basically a linear correction to those without the SOC term, $H_{0}$. Therefore, the spin-orbit splittings are proportional to $\lambda$ and the results presented in this work can be scaled accordingly.

\section{RESULTS}

We have calculated the electronic properties of many different chiral GNRs, varying their width and curvature. All calculations have been performed with the four-orbital parametrization explained in Sec. IV. Therefore, they show some differences with respect to the widely used one-orbital approach. $^{11}$ We present here the band structures for three representative chiral ribbons $[M(7,1), M(5,2)$, and $M(3,2)]$ and, for comparison, some zigzag GNRs of different widths. The $M(7,1)$ ribbon has a chiral angle $\theta=6.58^{\circ}$, close to the zigzag direction; the $M(5,2)$ has $\theta=16.02^{\circ}$; and the $M(3,2)$ GNR has $\theta=23.41^{\circ}$, closer to the armchair direction. The $M(7,1)$ and $M(3,2)$ GNRs have the same unit cell with 76 atoms, but with different orientation; i.e., the $\boldsymbol{H}$ and $\boldsymbol{T}$ vectors are interchanged. The $M(5,2)$ ribbon, in the intermediate chirality range, has a unit cell with 52 atoms. The $(5,2)$ edge has three armchair $(A)$ and two zigzag $(Z)$ units, so for the infinite system there are two possible arrangements of the armchair and zigzag units with the same edge vector. We choose the one with all zigzag units together, the $Z Z Z A A$. While the edge states in a semi-infinite graphene sheet or in very wide ribbons are the same irrespective of the sequence, for narrow ribbons some differences in the band structure of distinct edge arrangements may arise. 


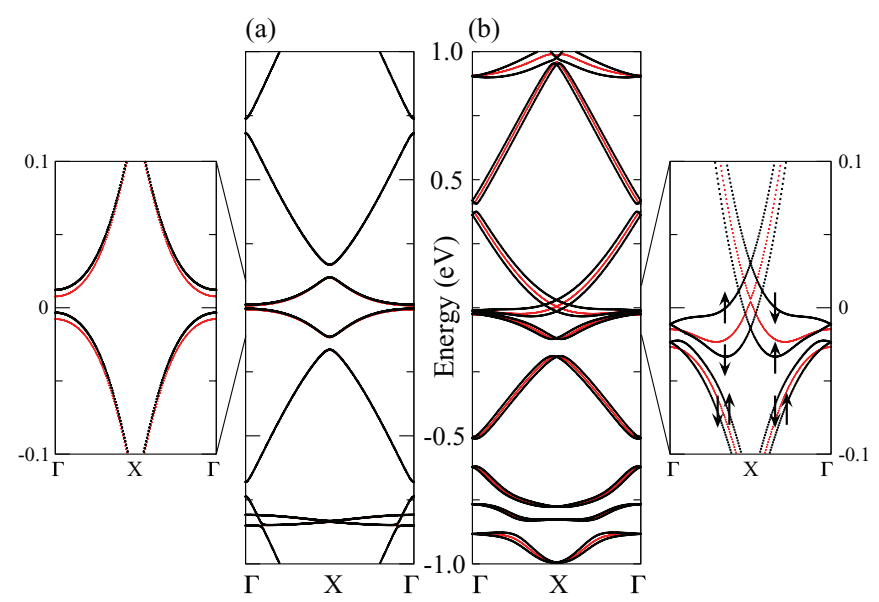

FIG. 3. (Color online) Electronic structure of the 1(7,1) GNR calculated with (black dots) and without (red and gray dots) SOC, considering (a) the four-orbital $s p^{3}$ basis set in a planar geometry and (b) the same $s p^{3}$ basis but for a curved geometry with $\varphi=2 \pi$. Zooms of the edge bands are included close to each panel.

\section{A. SOC and inversion symmetry}

\section{Curvature effects}

In systems with time-reversal and spatial inversion symmetry, spin-orbit interaction does not lift spin degeneracy, according to Kramers' theorem. If spatial inversion symmetry is not present, the states are spin split except in the $k$ points protected by time-reversal invariance. In symmetric-edge GNRs, spatial inversion symmetry is broken by curving the ribbon, as indicated in Fig. 2. The importance of the broken inversion symmetry is shown in Fig. 3, where the electronic structures of the $1(7,1)$ ribbon calculated with and without SOC terms are depicted for (a) the planar configuration and (b) the curved one with $\varphi=2 \pi$. This angle corresponds to a maximally curved geometry without overlapping the edges of the ribbon; it is equivalent to an open carbon nanotube with circumference equal to the width of the GNR. SOC effects are clear: all degeneracies, including spin, are lifted in the curved ribbon (b), while in the flat system (a) the bands remain spin degenerated. The only noticeable difference in the flat case is a small shift in the bands at the $\Gamma$ point, as can be observed in the zoom of Fig. 3. Otherwise, the effect of SOC is negligible. However, a large splitting is found in the curved ribbons, greater for the conduction bands. This is due to the interaction of edge states with higher-energy bands [see zoom in Fig. 3(b)], which in fact are completely hybridized due to curvature. Throughout most of the BZ, one of the spin-split bands has an upward shift in energy, whereas the other band undergoes a downshift. Thus, the bands without SOC mostly lie between the spin-orbit-split bands, as can be seen in Fig. 3(b), especially in the zoom.

Notice that GNRs can be made metallic because of the curvature, as seen in Fig. 3: the gap observed in the flat 1(7,1) ribbon (panel a) is still present at $\Gamma$ in the curved $(\varphi=2 \pi)$ geometry, but in this latter case the ribbon is metallic due to the band bending produced by curvature-induced hybridization (panel b).
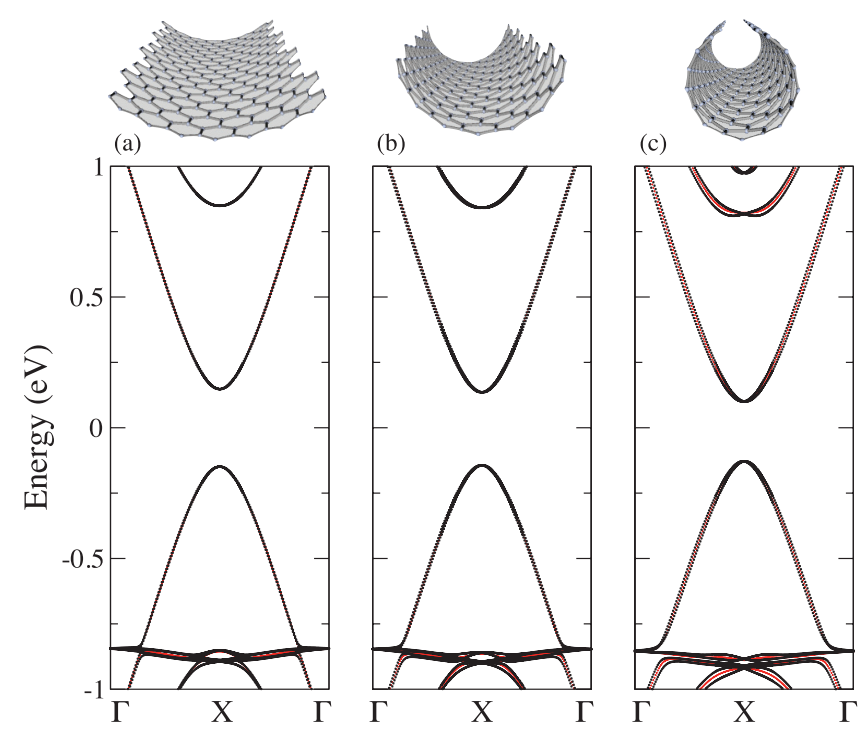

FIG. 4. (Color online) Electronic structure of the 1(3,2) GNR with different curvatures: (a) $\varphi=0.5 \pi$, (b) $\varphi=\pi$, and (c) $\varphi=1.8 \pi$. The bands with SOC are shown in black; bands without SOC are in red (gray).

Although the spin is no longer a good quantum number, the expectation value of the spin operator shows that in the flat geometry the total spin is normal to the ribbon. Curvature provokes the appearance of a small component in the in-plane direction which increases with curvature.

Figure 4 illustrates the effect of curvature. It presents the band structure of the $1(3,2)$ ribbon for three bending angles, namely, (a) $0.5 \pi$, (b) $1.2 \pi$, and (c) $1.8 \pi$, with (black dots) and without (red and gray dots) SOC. The curved geometries are shown above each band panel. In a wide $M(3,2)$ ribbon there are four edge bands at $0 \mathrm{eV}$ extending from $\frac{2}{3} \Gamma X$ to $X{ }^{8}$ In the case depicted in Fig. 4 a large gap opens between the occupied and unoccupied edge bands due to size effects, which we discuss later on. There is a general increase of the band splitting with growing curvature, as expected, due to the increment of the $\sigma-\pi$ hybridization produced in the curved ribbons, analogous to the effect predicted ${ }^{16,18}$ and experimentally measured in CNTs. ${ }^{21}$ Moreover, band splitting in GNRs is anisotropic, band and $k$ dependent, as also found in CNTs.

\section{Edge modification}

As discussed above, planar GNRs with dissimilar edges also lack inversion symmetry. Different edges can be achieved either by adding or removing atoms in a symmetric edge ribbon, or by altering the bond lengths at one edge of a symmetric GNR. We have explored the magnitude of this effect, calculating the changes in the band structure of a planar $1(5,2)$ modified ribbon. We have considered two types of modifications: two atoms of one edge have been removed, and the bond length of the zigzag atoms at one edge has been changed $10 \%$. With both modifications SOC breaks the spin degeneracy of the band structure; however, the splitting is two orders of magnitude smaller than that achieved by the effect 

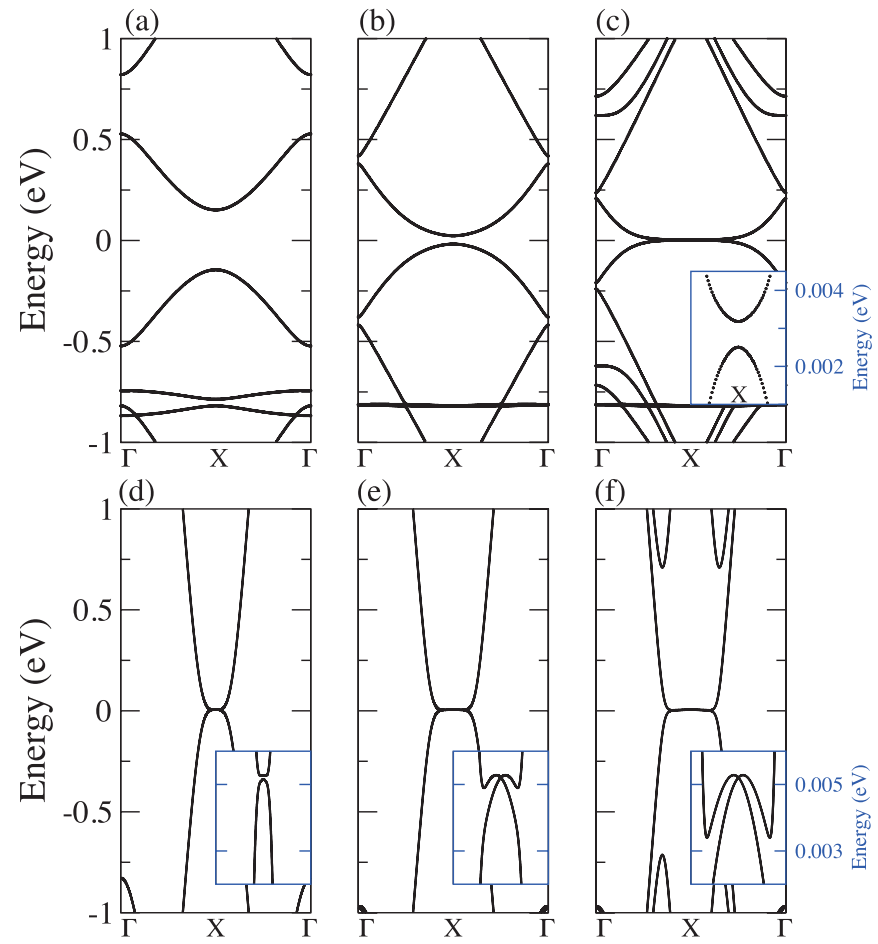

FIG. 5. (Color online) Band structures for flat ribbons calculated with the $s p^{3}$ basis set, including the SOC term for the following GNRs: (a) 1(5,2), (b) 2(5,2), (c) 4(5,2), (d) 2(1,0), (e) 4(1,0), and (f) $8(1,0)$. Insets: Zooms of the bands near $E_{F}$.

of curvature. Thus, in what follows, we concentrate on the curvature mechanism as a means to break inversion symmetry.

\section{B. SOC effects and width of the ribbon}

In order to explore the interplay of SOC and the width of the ribbon we consider first flat GNRs, since in curved geometries they could be masked by other effects, such as hybridization. We have performed calculations for different chiralities, verifying that there is a gap in planar chiral nanoribbons that decreases with increasing width. Figures 5(a)-5(c) demonstrate this effect for the $M(5,2)$ GNRs. The $1(5,2)$ ribbon has a substantial gap, around $0.4 \mathrm{eV}$, while the flat bands around $E_{F}$ for the 4(5,2) ribbon in panel c are clearly identified as edge bands for their dispersionless character near the BZ boundary X. Nonetheless, the gap can be discerned in the inset of Fig. 5(c), as stated above.

Comparison with high-symmetry zigzag ribbons of similar widths shows a striking difference. Figure 5 demonstrates that zigzag ribbons have a negligible energy gap, around $0.1 \mathrm{meV}$ for the narrowest case depicted [Fig. 5(d), $W=8.52 \AA$ ], while for the $1(5,2)$ ribbon of similar width $(8.87 \AA)$ the gap is around $0.4 \mathrm{eV}$.

The gaps between edge bands in chiral ribbons are due to the stronger coupling between edge states localized at each boundary. In zigzag ribbons the atoms at opposite edges belong to different sublattices, while in chiral ribbons boundary conditions at each edge mix the two sublattices, coupling the states located at the two edges. This results in a band gap due to quantum size effects, without invoking electron-electron interactions.

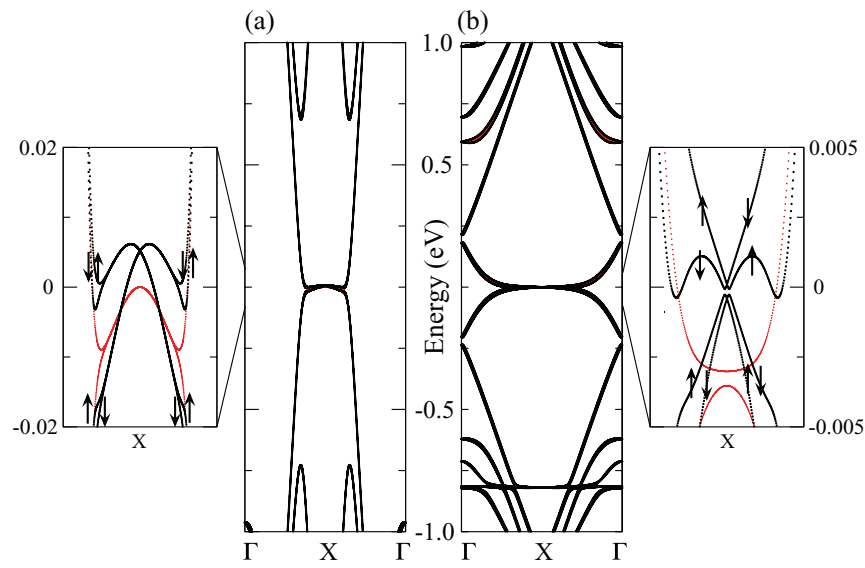

FIG. 6. (Color online) Band structures for curved ribbons calculated with the $s p^{3}$ basis set, for the following GNRs: (a) 8(1,0) and (b) 4(5,2). The bands with SOC are shown in black; bands without SOC are in red (gray). Insets: Zooms of the bands near $E_{F}$.

In flat ribbons, SOC induces a tiny shift of the energy bands; in zigzag ribbons, it turns the flat edge bands into dispersive ones. As inversion symmetry is preserved, all bands remain spin degenerated.

Now, we include curvature in order to enhance SOC effects and break spin degeneracy, as discussed in the previous section. For narrow chiral ribbons the gap due to quantum size effects is rather large, as can be seen in Figs. 5(a) and 5(b), so the effect of SOC is the aforementioned energy shift and, most importantly, the spin splitting of the bands. We focus on the widest ribbons, namely, $4(5,2)$ and the $8(1,0)$, with a smaller quantum size gap, and consider the same curvature radius for both ribbons, $R=6.274 \AA$, which yields an angle $\varphi=1.8 \pi$ and $1.73 \pi$, respectively. For these cases, the bands closer to $E_{F}$ are strongly deformed. These happen to be edge states, so their behavior gives rise to a more interesting situation than in the large gap ribbons, as is illustrated in Fig. 6.

Figure 6(a) shows the zigzag case, with a noticeable dispersion in the edge bands. The zoom shows that the bands with SOC are spin split, with a crossing point slightly shifted with respect to that of the bands without SOC. Figure 6(b) shows the chiral 4(5,2) GNR; here, besides the energy shift and spin splitting of the SOC bands, there is a slight displacement of the Fermi wave vector, which is no longer at X. Although small, SOC effects have important consequences for the transport properties of curved GNRs: spin-filtered channels arise due to the interplay of SOC and curvature, and for wider ribbons even chiral GNRs present these spin-filtered channels in the low-energy region.

\section{Chirality and spatial distribution of edge states}

Edge states are among the most important features of GNRs. ${ }^{9,10}$ It is interesting to explore how chirality affects the behavior of these states. For the sake of simplicity, we focus on flat geometries; generalization to curved geometries is straightforward. In Fig. 7 we present the electronic densities of edge states belonging to flat ribbons with different chiralities. The two states chosen correspond to the edge bands closer to the Fermi level, near the high-symmetry point to which 
(a)

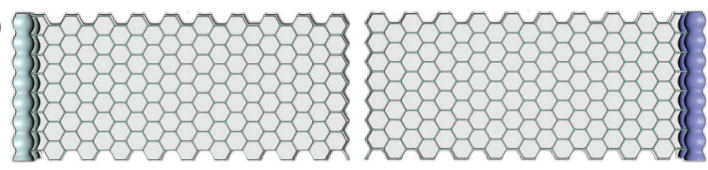

(b)

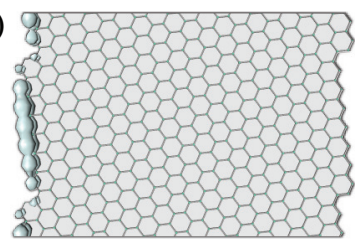

(c)

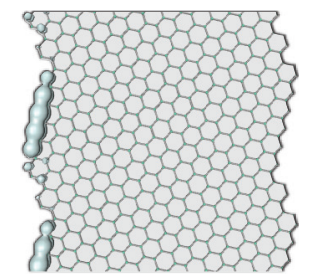

(d)

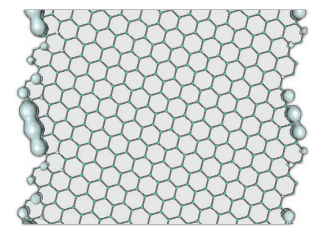

(e)
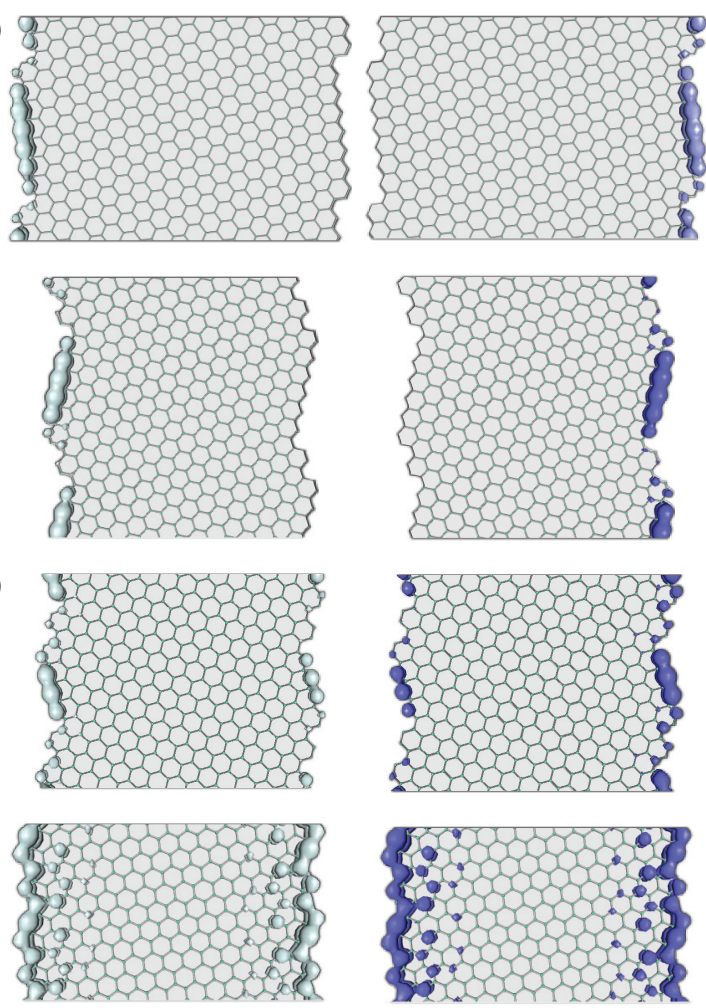

FIG. 7. (Color online) Probability densities corresponding to two states of the edge bands closer to $E_{F}$, near a high-symmetry point, for several flat ribbons. The spin polarization is plotted in white for spin up and blue (gray) for spin down. (a) Zigzag 10(1,0) ribbon, $k=0.95 \Gamma \mathrm{X}$. (b) Chiral $4(7,1)$ ribbon, $k=0.10 \Gamma \mathrm{X}$. (c) Chiral $1(7,2)$ ribbon, $k=0.95 \Gamma \mathrm{X}$. (d) Chiral 4(5,2) ribbon, $k=0.10 \Gamma \mathrm{X}$. (e) Chiral 2(3,2) ribbon, $k=0.95 \Gamma \mathrm{X}$.

the edge bands converge for large widths. Opposite spin polarizations are indicated with distinct colors; since we are dealing with flat ribbons, the spin direction is perpendicular to the plane of the ribbon. We choose a $k$ value slightly displaced from the symmetry point (either $\Gamma$ or the BZ boundary X) in order to avoid degeneracy due to time-reversal invariance and ribbons of similar width in order to compare size effects. In pure zigzag ribbons, edge states are spin filtered: each edge state has a well-defined spin orientation and it is located at one edge. This is illustrated in Fig. 7(a), which shows the square modulus of the wave functions for two edge states corresponding to a $10(1,0)$ zigzag nanoribbon, of width equal to $42.61 \AA$. These two edge states of zigzag ribbons live in opposite sublattices, and their probability density is mostly confined to the atoms with coordination number 2 , which constitute the geometrical edge. Figure 7(b) shows the density for two edge states of a $4(7,1)$ ribbon of width $42.89 \AA$, close in chirality to the zigzag case. Similarly to the zigzag ribbon, the density of each edge is mostly located in one sublattice. It is not homogeneously distributed, being mostly in atoms with coordination number 2 , although there is some appreciable weight in inner atoms close to the edge. For the $1(7,2)$ GNR (of width $34.88 \AA$ and $\theta=12.21^{\circ}$ ) [Fig. 7(c)], which has a larger chirality angle, the wave function extends more into inner atoms, especially close to the armchair part of the boundary.

For chiralities closer to the armchair, the states have a nonzero density at both edges: panel d shows the two states close to $E_{F}$ for a $4(5,2)$ ribbon of width $35.48 \AA$. These two states live at both edges simultaneously, with more inner atoms with nonzero density. This is more dramatic in panel e, which depicts the edge states for a $2(3,2)$ ribbon of width $37.40 \AA$. As its chirality is closer to the armchair case, edge states have a greater penetration into the inner part of the ribbon. In order to localize these states and obtain the quantum spin Hall phase, a larger value of the SOC constant $(\lambda \approx 4 \mathrm{eV})$ is needed. This behavior has been found for armchair ribbons within the Dirac model $^{31}$ and the ETB model. ${ }^{23}$ The SOC strength required increases with the chiral angle and decreases with the width of the ribbon.

In curved ribbons, edge states keep their localized character, even for the maximum curvature. Besides the spin splitting (Fig. 6), there are two main differences: the spatial localization length is larger for the curved ribbon than for the flat case, and the spin direction changes from the direction normal to the ribbon surface, acquiring a component in the ribbon plane, as previously reported. ${ }^{23,24}$

\section{DISCUSSION AND CONCLUSIONS}

Our results show that the relation between the QSH edge states of graphene nanoribbons and both the crystallographic orientation of the edges and the curvature of the GNR allows us to control the spin and spatial localization of the ribbon edge states. Consequently, this may allow us to achieve an efficient electrical control of spin currents and spin densities in GNRs. Taking into account both spin and valley degeneracy, Bloch states in graphene are fourfold degenerate. SOC splits them into two Kramers doublets and, as Kane and Mele predicted, ${ }^{12}$ this turns graphene into a topologically nontrivial material. In zigzag edge ribbons, it has already been shown that at each edge spin-up and spin-down electrons move in opposite directions. ${ }^{20,23}$ Since backscattering in a given edge requires the reversal of spin it cannot be induced by spin-independent scatterers. Accordingly, edge states in zigzag GNRs are topologically protected and hence the conductance of the edge states is quantized. However, in flat chiral-edge GNRs of finite width and for realistic values of the SOC strength, there is a non-zero probability of having electrons moving in opposite directions with the same spin polarization at a given edge (see Fig. 7). Therefore, intra-edge backscattering may occur, which affects the quantization of the conductance. In chiral-edge GNRs, spin reversal can be induced even by nonmagnetic disorder and thus edge states do not present a robust conductance quantization. The appearance of backscattering does depend on the chirality angle, increasing for angles approaching the armchair limit. On the other hand, curvature breaks the inversion symmetry of the ribbons and Bloch states are spin split. Electrons with the same spin and opposite propagating directions in a given edge have different energies. As a result, backscattering is not allowed, and in curved chiral-edge GNRs edge states behave 
as robust quantum channels. Therefore, these chiral ribbons present a magnetomechanical effect: upon curving the ribbon, the spin channels are split in energy, thus allowing for spatially separated spin currents.

Despite the weakness of SOC in these carbon systems-on the scale of a few meV-their effects in curved graphene and nanotubes are not negligible, due to the coupling of $\pi$ and $\sigma$ bands in curved geometries. Thus, although small, the effects discussed in this work may be physically relevant, and since in graphene the position of the Fermi level can be adjusted with external gate voltages the control of spin currents in GNRderived devices could be possible.

In summary, we have shown that, in the presence of spin-orbit interaction, curvature breaks spin degeneracy in graphene ribbons. Flat nanoribbons with symmetric edges, either chiral or achiral, have spin-degenerate bands. This is due to the existence of spatial inversion symmetry in flat ribbons, which is broken in the curved cases.

Furthermore, spin-orbit splitting is enhanced in curved ribbons due to the hybridization of the bands, absent in flat samples. Other mechanisms to break inversion symmetry, such as edge modification, are much less efficient to remove spin degeneracy.
We have also explored finite-size effects in GNRs. We find that narrow chiral ribbons present a sizable gap, despite their having a zigzag edge component, whereas in pure zigzag GNRs of similar width the gap is negligible. We relate this behavior to the boundary conditions in chiral edges, which mix the two sublattices at each edge. Finally, we have studied the chirality dependence on the spatial localization of edge states. In narrow chiral ribbons, edge states have a nonzero density at both edges simultaneously, due to edge coupling. Due to the sublattice mixing produced by the chiral boundary conditions, edge states have a larger penetration than those of achiral ribbons. For wider curved ribbons, they behave as spin-filtered states, being localized at one edge.

\section{ACKNOWLEDGMENTS}

H.S. gratefully acknowledges helpful discussions with J. E. Alvarellos. This work has been partially supported by the Spanish Ministries of Science and Innovation (MICINN) and Economy and Competitivity (MINECO) DGES under Grants No. MAT2009-14578-C03-03, No. PIB2010BZ-00512, No. FIS2010-21282-C02-02, No. FIS2011-23713, No. MAT201238045-C04-04, and No. FIS2012-33521.
${ }^{1}$ A. H. Castro Neto, F. Guinea, N. M. R. Peres, K. S. Novoselov, and A. K. Geim, Rev. Mod. Phys. 81, 109 (2009).

${ }^{2}$ D. V. Kosynkin, A. L. Higginbotham, A. Sinitskii, J. R. Lomeda, A. Dimiev, B. K. Price, and J. M. Tour, Nature (London) 458, 872 (2009).

${ }^{3}$ Y.-M. Lin, A. Valdes-Garcia, S. Han, D. B. Farmer, I. Meric, Y. Sun, Y. Wu, C. Dimitrakopoulos, A. Grill, P. Avouris, and K. Jenkins, Science 332, 1294 (2011).

${ }^{4}$ M. Fujita, K. Wakabayashi, K. Nakada, and K. Kusakabe, J. Phys. Soc. Jpn. 65, 1920 (1996).

${ }^{5}$ K. Wakabayashi, S. Okada, R. Tomita, S. Fujimoto, and Y. Natsume, J. Phys. Soc. Jpn. 79, 034706 (2010).

${ }^{6}$ Y. Niimi, T. Matsui, H. Kambara, K. Tagami, M. Tsukada, and H. Fukuyama, Phys. Rev. B 73, 085421 (2006).

${ }^{7}$ A. R. Akhmerov and C. W. J. Beenakker, Phys. Rev. B 77, 085423 (2008).

${ }^{8}$ W. Jaskólski, A. Ayuela, M. Pelc, H. Santos, and L. Chico, Phys. Rev. B 83, 235424 (2011).

${ }^{9}$ K. Wakabayashi, Y. Takane, M. Yamamoto, and M. Sigrist, Carbon 47, 124 (2009).

${ }^{10}$ K. Wakabayashi and S. Dutta, Solid State Commun. 152, 1420 (2012).

${ }^{11}$ O. V. Yazyev, R. B. Capaz, and S. G. Louie, Phys. Rev. B 84, 115406 (2011).

${ }^{12}$ C. L. Kane and E. J. Mele, Phys. Rev. Lett. 95, 226801 (2005).

${ }^{13}$ M. Z. Hasan and C. L. Kane, Rev. Mod. Phys. 82, 3045 (2010).

${ }^{14}$ W. Beugeling, N. Goldman, and C. M. Smith, Phys. Rev. B 86, 075118 (2012).

${ }^{15}$ T. Ando, J. Phys. Soc. Jpn. 69, 1757 (2000).

${ }^{16}$ L. Chico, M. P. López-Sancho, and M. C. Muñoz, Phys. Rev. Lett. 93, 176402 (2004).

${ }^{17}$ D. Huertas-Hernando, F. Guinea, and A. Brataas, Phys. Rev. B 74, 155426 (2006).
${ }^{18}$ L. Chico, M. P. López-Sancho, and M. C. Muñoz, Phys. Rev. B 79, 235423 (2009).

${ }^{19}$ W. Izumida, K. Sato, and R. Saito, J. Phys. Soc. Jpn. 78, 074707 (2009).

${ }^{20}$ L. Chico, H. Santos, M. C. Muñoz, and M. P. López-Sancho, Solid State Commun. 152, 1477 (2012).

${ }^{21}$ F. Kuemmeth, S. Ilani, D. C. Ralph, and P. L. McEuen, Nature (London) 452, 448 (2008).

${ }^{22}$ M. Zarea and N. Sandler, Phys. Rev. B 79, 165442 (2009).

${ }^{23}$ M. P. López-Sancho and M. C. Muñoz, Phys. Rev. B 83, 075406 (2011).

${ }^{24}$ D. Gosálbez-Martínez, J. J. Palacios, and J. Fernández-Rossier, Phys. Rev. B 83, 115436 (2011).

${ }^{25}$ H. Santos, L. Chico, and L. Brey, Phys. Rev. Lett. 103, 086801 (2009).

${ }^{26}$ C. Tao, L. Jiao, O. V. Yazyev, Y.-C. Chen, J. Feng, X. Zhang, R. B. Capaz, J. M. Tour, A. Zettl, S. G. Louie, H. Dai, and M. F. Crommie, Nat. Phys. 7, 616 (2011).

${ }^{27}$ R. Saito, G. Dresselhaus, and M. S. Dresselhaus, Physical Properties of Carbon Nanotubes (Imperial College Press, London, 1998).

${ }^{28}$ J. C. Charlier, X. Blase, and S. Roche, Rev. Mod. Phys. 79, 677 (2007).

${ }^{29}$ D. Tománek and S. G. Louie, Phys. Rev. B 37, 8327 (1988).

${ }^{30}$ H. Min, J. E. Hill, N. A. Sinitsyn, B. R. Sahu, L. Kleinman, and A. H. MacDonald, Phys. Rev. B 74, 165310 (2006).

${ }^{31}$ M. Zarea and N. Sandler, Phys. Rev. Lett. 99, 256804 (2007).

${ }^{32}$ S. Konschuh, M. Gmitra, and J. Fabian, Phys. Rev. B 82, 245412 (2010).

${ }^{33}$ G. Steele, F. Pei, E. Laird, J. Jol, H. Meerwaldt, and L. Kouwenhoven, Nature Communications 4, 1873 (2013).

${ }^{34}$ M. B. Lundeberg, R. Yang, J. Renard, and J. A. Folk, Phys. Rev. Lett. 110, 156601 (2013). 